\title{
Successive Vaccination and Difference in Immunity of a Delay SIR Model with a General Incidence Rate
}

\author{
Yongzhen Pei, ${ }^{1}$ Li Changguo, ${ }^{2}$ Qianyong Wu, ${ }^{1}$ and Yunfei $\mathrm{Lv}^{3}$ \\ ${ }^{1}$ School of Computer Science and Software Engineering, Tianjin Polytechnic University, Tianjin 300387, China \\ ${ }^{2}$ Department of Basic Science, Military Transportation University, Tianjin 300161, China \\ ${ }^{3}$ School of Science, Tianjin Polytechnic University, Tianjin 300387, China \\ Correspondence should be addressed to Yongzhen Pei; yongzhenpei@163.com
}

Received 28 December 2013; Revised 7 May 2014; Accepted 8 May 2014; Published 16 June 2014

Academic Editor: Kaifa Wang

Copyright (C) 2014 Yongzhen Pei et al. This is an open access article distributed under the Creative Commons Attribution License, which permits unrestricted use, distribution, and reproduction in any medium, provided the original work is properly cited.

A delay SIR epidemic model with difference in immunity and successive vaccination is proposed to understand their effects on the disease spread. From theorems, it is obtained that the basic reproduction number governs the dynamic behavior of the system. The existence and stability of the possible equilibria are examined in terms of a certain threshold condition about the basic reproduction number. By use of new computational techniques for delay differential equations, we prove that the system is permanent. Our results indicate that the recovery rate and the vaccination rate are two factors for the dynamic behavior of the system. Numerical simulations are carried out to investigate the influence of the key parameters on the spread of the disease, to support the analytical conclusion, and to illustrate possible behavioral scenarios of the model.

\section{Introduction}

The current threat of some new type diseases has raised our awareness that curbing the spread of some emerging and reemerging human diseases is of public health importance such as H1N1. This emerging disease, which was first reported in Mexico, spread very quickly, due to the travel of infected persons by airplanes, trains, and buses to some other regions. It continued to spread around the world and caused about 5000 deaths. In recent years, many mathematical models have been developed for the transmission dynamics of infectious diseases such as SARS, HIV/AIDS, measles, and smallpox ([1-6], to name a few, and the references therein). These models have provided understanding of the underlying mechanisms which influence the spread of diseases and suggested some control strategies. Moreover, to our knowledge, the first effective control strategy for the elimination of infectious diseases is obtaining immunity. It has been reported that "People's immunity to A/H1N1 flu virus is greater than previously thought after access vaccines. The WHO is working to give more nations access vaccines to fight the H1N1 flu pandemic."
Besides these above studies, many authors formulated and analyzed SIR epidemic models for the control of diseases [7-20]. In particular, some authors have studied the effects of vaccination on the spread of diseases [7-11]; others have studied the effects of treatment on the spread of diseases [12-15]. Gao et al. have proposed an epidemic model with density-dependent birth pulses and seasonal prevention [16]. Recently, some works have investigated permanent and temporary immunity [17-20]. However, in these SIR models, an unrealistic assumption is that all the rest of infected individuals acquire immunity besides death. Measles encephalitis in adults in $[21,22]$ shows that there is difference in immunity of infected individuals. That is, some infected individuals can acquire immunity after recovery, but some do not acquire immunity and can be infected once more. At the same time, vaccination is an important strategy for the elimination of infectious diseases [7-11].

Vaccinations have many types; impulsive vaccination and successive vaccination are two main policies. Successive vaccination is that people have been vaccinated at birth to protect themselves from disease; the studies can be found in 
$[23,24]$. Makinde in [23] studied a SIR model for the transmission dynamics of a childhood disease in the presence of a preventive vaccine and analyzed the vaccination reproductive number for disease control and eradication qualitatively. Impulsive vaccination (only at fixed time sequence we execute effectively the vaccination for the disease) is an important and effective strategy for the elimination of infectious diseases and has been studied in the literature. For example, see $[10,17,25-27]$. In above-mentioned papers, authors almost considered the vaccination of susceptible population. But, in fact, under a certain situation, the vaccine treatment also should be considered for the newborns of the susceptible, the exposed, and the removed. We find that there are few studies on the aspect of the vaccination of newborns. In this case, successive vaccination seems more reasonable than impulsive vaccination. Therefore, in this paper, a SIR model with difference in immunity and successive vaccination is considered.

As far as disease transmission is concerned, the incidence rate, defined as the rate of new infection, plays a very important role in modelling infectious diseases. Bilinear incidence rate $\beta S I$ in $[28,29]$ and standard incidence rates $\lambda S I / N$ in $[30,31]$ have often been used in epidemic models. However, it is unreasonable to consider the bilinear incidence rate (based on the law of mass action) as the number of susceptibles is large, owing to the number of susceptibles with which every infective contact within a certain time is limited. Standard incidence rate may be a good approximation if the number of available partners is large enough but it is not possible to make more contacts when the population $N$ is small. Combine the two previous approaches by assuming that if the number of available partners $N$ is low, the number of actual per capita partners is proportional to $N$, whereas if the number of available partners is large, there is a saturation effect which makes the number of actual partners constant. Considering this case, a saturation incidence rate of type $f(I) S$ with $f(I)=k I /(1+\alpha I)$ is being proposed in [32]. More general incidence rate used in the literature is the one for which $f(I)=k I^{l} /\left(1+\alpha I^{h}\right)[33,34]$, where $I^{l}$ measures the infection force of the disease and $f(I)=1 /\left(1+\alpha I^{h}\right)$ measures the inhibitory effect caused by behavioral changes. Note that if $f(I)$ is decreasing when $I$ is large, this may be interpreted as the fact that susceptibles tend to reduce their social contacts if the perceived number of infectives increases over a psychologically significant value. The above saturation incidence rate depends also on the size of the infectives $I$ termed as infectives-dependent. Particular examples of susceptibles-dependent incidence rate are $f(S)=k S /(1+\alpha S)$ [35]. Very general incidence rates which are not linear in $S$ are also used in Derrick and van den Driessche [36] $(f(S, I, N)=$ $I \Phi(S, I, N)$, where $N=S+I)$, Korobeinikov and Maini [37] $\left(f(S, I)=h_{1}(I) h_{2}(S)\right)$, and Moghadas and Alexander [38] $(f(S, I)=\beta(1+g(I, v)) I S)$.

Nie et al. [19] and Ji et al. [39] respectively considered a delay SIR epidemic model with nonlinear incidence rate and density-dependent birth and death rates. Motivated by the main idea described in $[6,39]$, in this paper, we consider a delay SIR model with difference in immunity and successive vaccination and an abstract incidence rate. The main difference between our study and those described in [6, 39 ] is the difference in immunity and successive vaccination and an abstract incidence rate. An abstract incidence rate of type $f(I) S$ is employed to model the spread of the disease which is propagated through the infective individuals, under a few biologically feasible assumptions upon $f(I)$.

In view of above facts, we will formulate a mathematical model in Section 2. We provide the region of biologically feasible solutions in Section 3. Then, we study the existence and stability of the steady states in the next section, analyze the permanence result in Section 5, and give some numerical simulations in Section 6. Lastly, we end the paper with a brief discussion of our results in Section 6.

\section{Model Formulation and Invariant Region}

In this section, we will present a delay SIR epidemic model with a general nonlinear incidence rate. The total population $N(t)$ is divided into three subclasses, namely, the susceptibles $S(t)$, the infectives $I(t)$, and the recovered individuals $R(t)$. Based on the SIR model in $[12,39]$, we considered following system:

$$
\begin{aligned}
& \frac{d S}{d t}= {\left[b-\frac{a r N}{K}\right] N-\beta e^{-d_{1} \tau} S f(I(t-\tau)) } \\
&-\left[d+\frac{(1-a) r N}{K}\right] S-\theta S+\mu_{1} I, \\
& \frac{d I}{d t}= \beta e^{-d_{1} \tau} f(I(t-\tau)) S \\
&-\left[d+\frac{(1-a) r N}{K}\right] I-\mu_{1} I-e^{-d_{1} \omega} \delta I(t-\omega), \\
& \frac{d R}{d t}= e^{-d_{1} \omega} \delta I(t-\omega)-\left[d+\frac{(1-a) r N}{K}\right] R+\theta S, \quad I(0)=\varphi(\theta) \geq 0, \\
& \quad N(0)=S_{0}>0, \quad \forall \theta \in[-\bar{\tau}, 0], R(0)=R_{0} \geq 0
\end{aligned}
$$

where $\bar{\tau}=\max \{\tau, \omega\}$ and $\varphi \in C([-\bar{\tau}, 0], R)$. We give the following useful assumptions.

(1) There are no disease induced deaths, and all the newborns are susceptible.

(2) $f(I)$ is the nonlinear incidence rate satisfying the following assumptions:

$$
\begin{gathered}
f(0)=0, \quad f^{\prime}(I)>0, \quad f^{\prime \prime}(I)<0, \\
\lim _{t \rightarrow \infty} f(I)=c<+\infty .
\end{gathered}
$$

(3) The force of infection at any time $t$ is dominated by $\beta e^{-d_{1} \tau} S(t) f(I(t-\tau))$, where $\tau$ is incubation period and $0<e^{-d_{1} \tau} \leq 1$ represents the survival probability of individuals in the population after time $\tau$ [20]. It is also assumed that $d_{1} \leq d$ in $[-\tau, 0]$, where $d$ is the death rate and $d_{1}$ is the death rate in the time interval $[-\tau, 0]$. 
(4) The parameters $a$ are a convex combination constant, $r=b-d>0$ is the intrinsic growth rate $(b$ is the birth rate), and $K>0$ is the carrying capacity of the population. The term $(b-(\operatorname{arN}(t) / K))$ has a densitydependent per capita birth rate and the term $(d+((1-$ a) $r N(t) / K)$ ) has a density-dependent per capita death rate [39].

(5) For $0<a<1$, the birth and death rates are consistent with the limited resources associated with density dependence. The birth rate is density independent when $a=0$ and the death rate is density independent when $a=1$. Thus, the spread of the disease (animals such as rodents, etc.) is assumed to be governed by the following system of logistic equations with time delay.

(6) The total population is assumed to be large enough to be adequately described by a deterministic model and is divided into compartments based on the disease status [40].

(7) The successive vaccination rate $\theta$ is positive. The positive constant $\mu_{1}$ is the recovery rate of the infectious individuals from compartment $I$ to $S$. The parameters $\beta$ are the effective per capita contact rate constant of infected individuals. The parameters $\delta$ are the recovery rate of infected individuals.

(8) Models are formulated as functional differential and/or integral equations when time delay is included [40]. Ours follows the former with the assumption that the $I$-equation satisfies a certain integral condition [41].

Models with multiple delays are not common, but few authors have in the past considered these-Beretta et al. [42]to name but a few. Since $N(t)=S(t)+I(t)+R(t)$, thus the governing equation (1) can be rewritten as

$$
\begin{gathered}
\frac{d N}{d t}=r\left[1-\frac{N}{K}\right] N, \\
\frac{d I}{d t}=\beta e^{-d_{1} \tau}(N-I-R) f(I(t-\tau)) \\
-\left[d+\frac{(1-a) r N}{K}\right] I-\mu_{1} I-e^{-d_{1} \omega} \delta I(t-\omega), \\
\frac{d R}{d t}=e^{-d_{1} \omega} \delta I(t-\omega) \\
-\left[d+\frac{(1-a) r N}{K}\right] R+\theta(N-I-R) .
\end{gathered}
$$

Let $\bar{\tau}=\max (\omega, \tau)$. Then (3) satisfies the following initial conditions

$$
\begin{array}{r}
N(0)=S_{0}>0, \quad I(0)=\varphi(\theta) \geq 0, \\
\forall \theta \in[-\bar{\tau}, 0], \quad R(0)=R_{0} \geq 0 .
\end{array}
$$

In this paper, we will consider two different delays $\tau, \omega$ which are important parameters on the dynamic behavior. So, the present study is continuation of the previous work $\tau=\omega$ by Naresh et al. [43].

Lemma 1. All solutions of the model system (3) starting in $R_{+}^{3}$ are bounded and eventually enter the compact attracting set

$$
\Phi=\left\{(S, I, R) \in R_{+}^{3}: S(t)+I(t)+R(t)=N(t) \leq K\right\} .
$$

Lemma 2. Let the initial data be $N(0)=S_{0}>0, I(0)=$ $I_{0}(u) \geq 0$, for all $u \in[-\bar{\tau}, 0]$, with $I_{0}(0)>0, R(0)=R_{0} \geq$ 0 . Then, the solution $(S(t), I(t), R(t))$ of the model remains positive for all time $t>0$.

Lemma 3 (see [44]). For the characteristic equation in the form $p(\lambda)+q(\lambda) e^{-r \lambda}=0$, where $p$ and $q$ are polynomials with real coefficients and $r>0$ is the delay, suppose

(a) $p(\lambda) \neq 0, R(\lambda)>0$;

(b) $|q(i y)|<|p(i y)| ; 0 \leq y<\infty$;

(c) $\lim _{|\lambda| \rightarrow \infty, R(\lambda) \geq 0}|q(\lambda) / p(\lambda)|=0$.

Then $R(\lambda)<0$ for every root $\lambda$ and all $r>0$.

\section{Equilibrium and Stability Analysis}

In this section, we focus on the existence and local stability of equilibria. Let the right-hand side of equalities in model (3) be zero. Then, there are two equilibria; namely,

(i) $\left.E_{0}=(K, 0, p), p=K \theta /[d+(1-a) r+\theta]\right)$, disease-free equilibrium;

(ii) $E^{*}=\left(N^{*}, I^{*}, R^{*}\right)$, endemic equilibrium,

where the values of $N^{*}, I^{*}$, and $R^{*}$ are given in Section 3.2.

3.1. Community Matrix. Firstly, after computing the Jacobian or community matrix of model (3) at point $(N, I, R)$, the characteristic equation is given by

$$
\left|\begin{array}{ccc}
r-\frac{2 r}{K} N-\lambda & 0 & 0 \\
\beta e^{-d_{1} \tau} f(I)-\frac{(1-a) r}{K} I & m e^{-\left(d_{1}+\lambda\right) \tau}-\delta e^{-\left(d_{1}+\lambda\right) \omega}-n-\lambda & \beta e^{-d_{1} \tau} f(I) \\
\theta-\frac{(1-a) r}{K} R & \delta e^{-\left(d_{1}+\lambda\right) \omega}-\theta & -\left[d+\frac{(1-a) r}{K} N+\theta\right]-\lambda
\end{array}\right|=0
$$


where $m=\beta(N-I-R) f^{\prime}(I), n=\beta e^{-d_{1} \tau} f(I)+d+((1-$ a) $r / K) N+\mu_{1}$.
Now, we analyze the equilibria stability of system (3). Computing the Jacobian of system (3) evaluated at $E_{0}$, one gets the following matrix

$$
J\left(E_{0}\right)=\left(\begin{array}{ccc}
-r-\lambda & 0 & 0 \\
0 & a_{22}-\lambda & 0 \\
\frac{(d+\theta) \theta}{d+(1-a) r+\theta} & \delta e^{-\left(d_{1}+\lambda\right) \tau}-\theta & -[d+(1-a) r+\theta]-\lambda
\end{array}\right)
$$

where

$$
\begin{aligned}
a_{22}= & \beta(K-p) f^{\prime}(0) e^{-\left(d_{1}+\lambda\right) \tau} \\
& -\delta e^{-\left(d_{1}+\lambda\right) \omega}-\left[d+(1-a) r+\mu_{1}\right] .
\end{aligned}
$$

Denote

$$
A=\beta(K-p) f^{\prime}(0), \quad C=d+(1-a) r+\mu_{1} ;
$$

then

$$
a_{22}=A e^{-\left(d_{1}+\lambda\right) \tau}-\delta e^{-\left(d_{1}+\lambda\right) \omega}-C .
$$

Denote

$$
h(\lambda)=A e^{-\left(d_{1}+\lambda\right) \tau}-\delta e^{-\left(d_{1}+\lambda\right) \omega}-C-\lambda .
$$

The eigenvalues of the system (3) about the steady state $E_{0}$ are $\lambda_{1}=-r, h(\lambda)=0$ and $\lambda_{3}=-[d+(1-a) r+\theta]$. All the parameters of the model are assumed to be nonnegative. Therefore, $\lambda_{1}$ and $\lambda_{3}$ are negative. Next, we discuss the roots of $h(\lambda)=0$ in five cases.

Case 1. For $\tau=\omega \neq 0$, from the second equation of the system (3), we can get the following.

Proposition 4. For $\tau=\omega>0, R(\lambda)<0$ for every root $\lambda$ of $h(\lambda)=0$ when

$$
(A-\delta) e^{-d_{1} \tau}<C .
$$

Proof. From the above analysis, $\lambda_{2}$ satisfies the following characteristic equation:

$$
g(\lambda)=(A-\delta) e^{-\left(d_{1}+\lambda\right) \tau}-C-\lambda=0 .
$$

(1) Clearly, $\lambda=0$ is not a root of $g(\lambda)=0$.

(2) From the fact that $g(0)<0, g^{\prime}(\lambda)<0$ for $\lambda>0$, it is obtained that $g(\lambda)=0$ has no positive real root.

(3) It is sufficient to show that $g(\lambda)=0$ does not admit a purely imaginary root. In fact, if $\lambda=i v(v>0)$ is a root of $(g(\lambda)=0)$, then by separating the real part, one gets

$$
(A-\delta) e^{-d_{1} \tau} \cos (v \tau)=C .
$$

Together with the condition of Proposition 4, we have

$$
\cos (v \tau)>1
$$

This is impossible.

(4) It is easy to show that $g(\lambda)=0$ has no imaginary root whose real part is positive. Otherwise, there is an imaginary $\operatorname{root} \lambda=u+i v$ with $u>0$. Without any loss of generality, we consider $v>0$. Then, we take the real and imaginary parts of $g(\lambda)=0$; namely,

$$
(A-\delta) e^{-\left(d_{1}+u\right) \tau} \cos (v \tau)=C+u .
$$

Combined with (9), we have

$$
C>(A-\delta) e^{-d_{1} \tau}>(A-\delta) e^{-\left(d_{1}+u\right) \tau} \cos (v \tau)=C+u
$$

This is a contradiction which implies that all eigenvalues roots of $g(\lambda)$ have negative real parts. Therefore, the disease-free equilibrium of the system (3) is locally asymptotically stable when (9) holds. The proof is completed.

Case 2. For $\tau \neq 0, \omega=0$, by the same way as in Case 1 , one gets the following.

Proposition 5. For all $\tau \neq 0, \omega=0, R(\lambda)<0$ for every root $\lambda$ of $h(\lambda)=0$ when

$$
A e^{-d_{1} \tau}-\delta<C
$$

Case 3. For $\omega \neq 0, \tau=0$, one gets the following.

Proposition 6. For $\omega \neq 0, \tau=0, R(\lambda)<0$ for every root $\lambda$ of $h(\lambda)=0$ when

$$
\delta e^{-d_{1} \omega}<|A-C|
$$

Proof. By the fact that $h(\lambda)=0$ is equivalent to $p(\lambda)+$ $q(\lambda) e^{-\lambda \omega}=0$ with $p(\lambda)=A-C-\lambda, q(\lambda)=-\delta e^{-d_{1} \omega}$.

(i) Suppose $\lambda=u+i v(u>0)$. Then, $p(\lambda)=A-C-u-$ $i v \neq 0$.

(ii) By $|q(i v)|=\delta e^{-d_{1} \omega},|p(i v)|=\mid A-C-$ $i v \mid=\sqrt{(A-c)^{2}+v^{2}}$, together with the condition of Proposition 6, we know that $|q(i v)|<|p(i v)|$. 
(iii) Suppose $\lambda=u+i v,(u>0)$. Then,

$$
\begin{gathered}
\lim _{u^{2}+v^{2} \rightarrow+\infty}\left|\frac{q(\lambda)}{p(\lambda)}\right|=\delta e^{-d_{1} \omega}, \\
\lim _{u^{2}+v^{2} \rightarrow+\infty} \frac{1}{\sqrt{(A-C-u)^{2}+v^{2}}}=0 .
\end{gathered}
$$

Then, using Lemma 3, we have $R(\lambda)<0$ for all $\omega$.

Case 4. For $\omega>\tau>0$, let $\varepsilon=\omega-\tau$. Then, $\omega=\tau+\varepsilon$. For fixed $\tau$,

$$
h(\lambda)=A e^{-\left(d_{1}+\lambda\right) \tau}-\delta e^{-\left(d_{1}+\lambda\right)(\tau+\varepsilon)}-C-\lambda .
$$

Let $\lambda=u+i v(u>0)$. Then, we take the real and imaginary parts of $h(\lambda)=0$; namely,

$$
\begin{gathered}
A e^{-\left(d_{1}+u\right) \tau} \cos (v \tau)-\delta e^{-\left(d_{1}+u\right)(\tau+\varepsilon)} \cos (v(\tau+\varepsilon)) \\
=C+u, \\
-A e^{-\left(d_{1}+u\right) \tau} \sin (v \tau)-\delta e^{-\left(d_{1}+u\right)(\tau+\varepsilon)} \sin (v(\tau+\varepsilon))=v .
\end{gathered}
$$

Sum of squares of the above equalities is

$$
\begin{aligned}
& A^{2} e^{-2\left(d_{1}+u\right) \tau}+\delta^{2} e^{-2\left(d_{1}+u\right)(\tau+\varepsilon)} \\
& \quad-2 A \delta e^{-\left(d_{1}+u\right)(2 \tau+\varepsilon)} \cos (v \varepsilon)-(C+u)^{2}-v^{2}=0 .
\end{aligned}
$$

Then, we have

$$
\left.\frac{\partial u}{\partial \varepsilon}\right|_{\varepsilon=0}=\frac{\delta\left(u+d_{1}\right)(A-\delta)}{(A-\delta)^{2} \tau+(C+u) e^{2 \tau(u+\delta)}}
$$

Obviously, $\partial u /\left.\partial \varepsilon\right|_{\varepsilon=0}<0$ when $A-\delta<0$. Combined with $(A-\delta) e^{-d_{1} \tau}<A e^{-d_{1} \tau}-\delta e^{-d_{1} \omega}$, we have the following.

Proposition 7. For $\omega>\tau>0, R(\lambda)<0$ for every root $\lambda$ of $h(\lambda)=0$ when

$$
A e^{-d_{1} \tau}-\delta e^{-d_{1} \omega}<C, \quad A-\delta<0, \quad 0<\omega-\tau \ll 1 .
$$

Case 5. For $0<\tau<\omega$, in the same way as in Case 4 , we have the following.

Proposition 8. For $0<\tau<\omega, R(\lambda)<0$ for every root $\lambda$ of $h(\lambda)=0$ when

$$
A e^{-d_{1} \tau}-\delta e^{-d_{1} \omega}<C, \quad A-\delta>0, \quad 0<\tau-\omega \ll 1 .
$$
ing.

From what has been discussed above, we get the follow-

Theorem 9. The disease-free equilibrium of the system (3) is locally asymptotically stable if one of the following conditions holds.
(a) $\tau=\omega \neq 0,(A-\delta) e^{-d_{1} \tau}<C$.
(b) $\tau \neq 0, \omega=0, A e^{-d_{1} \tau}-\delta<C$.
(c) $\tau=0, \omega \neq 0, \delta e^{-d_{1} \omega}<|A-C|$.
(d) $A e^{-d_{1} \tau}-\delta e^{-d_{1} \omega}<C, A-\delta<0,0<\omega-\tau \ll 1$.
(e) $A e^{-d_{1} \tau}-\delta e^{-d_{1} \omega}<C, A-\delta>0,0<\tau-\omega \ll 1$.

3.2. Existence of Endemic Equilibrium. Thus, by Theorem 9, we may define the basic reproduction number as

$$
R_{0}=\frac{A e^{-d_{1} \tau}-\delta e^{-d_{1} \omega}}{C} .
$$

This threshold $R_{0}$ defines the average number of secondary infections generated by a typical infectious individual in a completely susceptible population in a steady demographic state.

In Theorem 9, we have already shown that the system (3) has an infection-free steady state which is locally asymptotically stable under condition $R_{0}<1$. The disease-free equilibrium is unstable when $R_{0}>1$, and the system (3) has a nontrivial endemic equilibrium $E^{*}=\left(N^{*}, I^{*}, R^{*}\right)$ when $R_{0}>1$. From (3),

$$
\begin{gathered}
N^{*}=K>0, \\
R^{*}=\frac{\delta e^{-d_{1} \omega}-\theta}{d+(1-a) r+\theta} I^{*} \\
+\frac{K \theta}{d+(1-a) r+\theta} \doteq q I^{*}+p,
\end{gathered}
$$

where $q=\left(e^{-d_{1} \omega} \delta-\theta\right) /(d+(1-a) r+\theta)$. Substituting these values of $N^{*}$ and $R^{*}$ in the second equation of (3), we get the following equation for $I$ :

$$
G(I)=\beta e^{-d_{1} \tau}(K-(1+q) I-p) f(I)-\left[C+\delta e^{-d_{1} \omega}\right] I .
$$

Obviously, $I=0$ is one of the roots of (29) as $f(0)=0$. Therefore, to exclude that root, choose

$$
H(I)=\beta e^{-d_{1} \tau}(K-(1+q) I-p) \frac{f(I)}{I}-\left[C+\delta e^{-d_{1} \omega}\right] .
$$

It can easily be seen that the function $H(I)$ is negative for large positive $I$; that is,

$$
H(K)=-\beta e^{-d_{1} \tau}(K q+p) \frac{f(k)}{K}-\left[C+\delta e^{-d_{1} \omega}\right]<0 .
$$

Next, we determine the sign of its derivative

$$
\begin{aligned}
H^{\prime}(I)= & \beta e^{-d_{1} \tau}(K-p) \frac{f^{\prime}(I) I-f(I)}{I^{2}} \\
& -\beta e^{-d_{1} \tau}(1+q) f^{\prime}(I) .
\end{aligned}
$$

It can easily be seen that $K>p$. In addition, from the properties of the function $f(I)$, in particular from $f(0)=0$ and $f^{\prime \prime}(0)<0$, it follows that $f(I)-f^{\prime}(I) I>0$, and consequently $H^{\prime}(I)<0$ for all $I>0$. Therefore, for a positive root of $H(I)=0$ to exist, $H(I)$ has to satisfy $H(0)>0$; that is,

$$
\begin{aligned}
H(0) & =A e^{-d_{1} \tau}-\delta e^{-d_{1} \omega}-C \\
& =\left(\frac{A e^{-d_{1} \tau}-e^{-d_{1} \omega} \delta}{C}-1\right) C \\
& =\left(R_{0}-1\right) C .
\end{aligned}
$$


Hence, one needs the requirement that $R_{0}>1$ to ensure the existence of the endemic equilibrium. From the above analysis, we have the following theorem.
Theorem 10. The system (3) has a nontrivial endemic equilibrium $E^{*}=\left(N^{*}, I^{*}, R^{*}\right)$ when $R_{0}>1$.

3.3. Local Stability of the Endemic Equilibrium. In this section, we analyze the local stability of the endemic equilibrium $E^{*}$ for $\tau=\omega$. Its characteristic equation is given by

$$
\left|\begin{array}{ccc}
-r-\lambda & 0 & 0 \\
\beta e^{-d_{1} \tau} f\left(I^{*}\right)-\frac{(1-a) r I^{*}}{K} & m e^{-\left(d_{1}+\lambda\right) \tau}-n-\lambda & \beta e^{-d_{1} \tau} f\left(I^{*}\right) \\
\theta-\frac{(1-a) r R^{*}}{K} & \delta e^{-\left(d_{1}+\lambda\right) \tau}-\theta & -[d+(1-a) r+\theta]-\lambda
\end{array}\right|=0
$$

where $m=\beta\left(K-I^{*}-R^{*}\right) f^{\prime}\left(I^{*}\right)-\delta, n=\beta e^{-d_{1} \tau} f\left(I^{*}\right)+d+$ $(1-a) r+\mu_{1}$.

The Jacobin matrix leads to the characteristic equation

$$
(\lambda+r)\left[\lambda^{2}+m_{1} \lambda+m_{0}+\left(n_{1} \lambda+n_{0}\right) e^{-\lambda \tau}\right]=0,
$$

where

$$
\begin{aligned}
m_{1}= & \beta e^{-d_{1} \tau} f\left(I^{*}\right)+2 d+2(1-a) r+\theta+\mu_{1}>0, \\
m_{0}= & {\left[\beta e^{-d_{1} \tau} f\left(I^{*}\right)+d+(1-a) r+\mu_{1}\right] } \\
& \times(d+(1-a) r+\theta)+\left(d+(1-a) r+\mu_{1}\right) \theta>0, \\
& n_{1}=-\left[\beta f^{\prime}\left(I^{*}\right)\left(K-I^{*}-R^{*}\right)-\delta\right] e^{-d_{1} \tau}, \\
n_{0}= & {\left[\beta f^{\prime}\left(I^{*}\right)\left(K-I^{*}-R^{*}\right)-\delta\right][d+(1-a) r+\theta] } \\
& \times e^{-d_{1} \tau}+\delta f\left(I^{*}\right) e^{-2 d_{1} \tau} .
\end{aligned}
$$

Since all the model parameters are assumed to be nonnegative, it follows that one eigenvalue is negative; that is, $\lambda_{1}=-r$. Thus, the stability of $E^{*}$ depends on the roots of the quasipolynomial

$$
\lambda^{2}+m_{1} \lambda+m_{0}+\left(n_{1} \lambda+n_{0}\right) e^{-\lambda \tau}=0
$$

We note that $m_{1}>0$ and $m_{0}>0$, whereas $n_{1}$ and $n_{0}$ may be positive or negative. For $\tau=0$, we state the following results that follow directly from (39). The endemic steady state is locally asymptotically stable if the following conditions hold:

$$
\begin{aligned}
& \beta f\left(I^{*}\right)+2 d+2(1-a) r+\theta+\mu_{1}+\delta \beta f^{\prime}\left(I^{*}\right) \\
& >\beta\left(K-I^{*}-R^{*}\right) f^{\prime}\left(I^{*}\right), \\
& {\left[\beta f\left(I^{*}\right)+d+(1-a) r+\mu_{1}+\delta-\beta\left(K-I^{*}-R^{*}\right) f^{\prime}\left(I^{*}\right)\right]} \\
& \quad \times[d+(1-a) r+\theta]+f\left(I^{*}\right) \delta>0 .
\end{aligned}
$$

The main purpose of this paper is to study the stability behavior of $E^{*}$ in the case $\tau \neq 0$. Obviously, in $(\eta>0)$ is the root of (29) if and only if $\eta$ satisfies

$$
-\eta^{2}+m_{1} i \eta+m_{0}=-\left(n_{1} i \eta+n_{0}\right)(\cos \eta \tau-i \sin \eta \tau) .
$$

Separating the real and imaginary parts, we have

$$
\begin{gathered}
-\eta^{2}+m_{0}=-n_{0} \cos \eta \tau-n_{1} \eta \sin \eta \tau, \\
m_{1} \eta=-n_{1} \eta \cos \eta \tau+n_{0} \sin \eta \tau .
\end{gathered}
$$

Eliminating $\tau$ by squaring and adding (39) and (40), we obtain a polynomial in $\eta$ as

$$
\eta^{4}+\left(m_{1}^{2}-n_{1}^{2}-2 m_{0}\right) \eta^{2}+m_{0}^{2}-n_{0}^{2}=0 .
$$

Suppose that the conditions

$$
m_{1}^{2}>n_{1}^{2}+2 m_{0}, \quad m_{0}^{2}>n_{0}^{2}
$$

hold for all $\tau \geq 0$. Then, the infected steady state of the system (3) is locally asymptotically stable.

Theorem 11. For $\tau=\omega$, if $R_{0}>1$, then the endemic equilibrium of the system (3) is locally asymptotically stable, when conditions $\left(\mathrm{H}_{1}\right)-\left(\mathrm{H}_{3}\right)$ hold.

Corollary 12. For $\tau=\omega$, if $\mu_{1}<\mu_{1}^{*}$ or $\theta<\theta^{*}$, then the endemic equilibrium of the system (3) is locally asymptotically stable, when conditions $\left(H_{1}\right)-\left(H_{3}\right)$ hold.

\section{Permanence}

In this section, we investigate a permanence result [5]. The following is our main result of this paper. We will give the following result by using some techniques given in $[8,11]$. The proof of the permanence with nonlinear incidence is a daunting task. Consequently, for simplicity and mathematical convenience, let us choose a linear incidence rate $f(I)=$ I. The result holds with the nonlinear incidence, as shown numerically, but the algebraic proof is long and tedious, and the conditions to impose on some of the parameters may be very restrictive. Now, let us firstly give the following theorem. 
Theorem 13. If $R_{0}>1$ holds, then the system (1) with $\tau=\omega$ is permanent; that is, there are positive constants $c_{i}(i=1,2,3)$ such that

$$
\begin{aligned}
& c_{1}<\lim _{t \rightarrow \infty} \inf S(t) \leq \lim _{t \rightarrow \infty} \sup S(t) \leq K, \\
& c_{2}<\lim _{t \rightarrow \infty} \inf I(t) \leq \lim _{t \rightarrow \infty} \sup I(t) \leq K, \\
& c_{3}<\lim _{t \rightarrow \infty} \inf R(t) \leq \lim _{t \rightarrow \infty} \sup R(t) \leq K
\end{aligned}
$$

hold for any solution of (1) with $\left(\phi_{1}(\theta), \phi_{2}(\theta), \phi_{3}(\theta)\right)$ in the interior of $\Phi$ for all $\theta \in[-\tau, 0]$. In fact, $c_{i}(i=1,2,3)$ can be chosen explicitly as

$$
\begin{gathered}
c_{1}=\frac{(b-a r) K}{\beta K e^{-d_{1} \tau}+d+(1-a) r+\theta}, \\
c_{2}=I^{*} e^{-\left(d+(1-a) r+\mu_{1}+e^{-d_{1} \tau} \delta\right)}, \\
c_{3}=\frac{\delta e^{-d_{1} \tau} c_{2}+\theta c_{1}}{d+(1-a) r} .
\end{gathered}
$$

Proof. Note that $0<N(t)<K$ for all $t \geq 0$ and that $\lim _{t \rightarrow \infty} N(t)=K$. It is easy to see that $\lim _{t \rightarrow \infty} \inf S(t) \geq c_{1}$. In fact, let $\epsilon<K$ be arbitrary. Choose $T_{1}>\tau$ so large that $N(t)>K-\epsilon$ for $t>T_{1}$. We have the following inequality:

$$
\begin{aligned}
\dot{S}(t)>- & {\left[\beta K e^{-d_{1} \tau}+d+(1-a) r+\theta\right] S(t) } \\
& +(b-a r)(K-\epsilon),
\end{aligned}
$$

for all $t \geq T_{1}$, which implies that

$$
\liminf _{t \rightarrow \infty}(t) \geq \frac{(b-a r)(K-\epsilon)}{\beta K e^{-d_{1} \tau}+d+(1-a) r+\theta} .
$$

Note that $\epsilon$ may be arbitrarily small so that $\lim _{t \rightarrow \infty} \inf S(t) \geq$ $c_{1}$.

Next, we will show $\lim _{t \rightarrow \infty} \inf I(t) \geq c_{2}$. For any $\xi$ : $0<\xi<1$, we see the inequality $S^{*}<[(b-a r) K+$ $\left.\mu_{1} I^{*}\right] /\left(\beta e^{-d_{1} \tau} \xi I^{*}+d+(1-a) r+\theta\right)$. There exist sufficiently large $\rho \geq 1$ and sufficiently small $\epsilon$ such that $S^{*}<\{[(b-$ ar $\left.\left.)(K-\epsilon)+\mu_{1} I^{*}\right] /\left(\beta e^{-d_{1} \tau} \xi I^{*}+d+(1-a) r+\theta\right)\right\}(1-$ $\left.e^{-\left(\beta e^{-d_{1} \tau} \xi I^{*}+d+(1-a) r+\theta\right) \rho \tau}\right) \equiv S^{\Delta}$. We show that $I\left(t_{0}\right)>q I^{*}$ for some $t_{0} \geq \rho \tau$. In fact, if not, it follows from the first equation of (1) that, for all $t \geq \rho \tau+\tau \geq T_{1}+\tau$,

$$
\begin{aligned}
\dot{S}(t) \geq & (b-a r)(K-\epsilon)+\mu_{1} I^{*} \\
& -\left[\beta e^{-d_{1} \tau} \xi I^{*}+d+(1-a) r+\theta\right] S(t) .
\end{aligned}
$$

Hence, for $t \geq \rho \tau+\tau$,

$$
\begin{aligned}
S(t) \geq & e^{-\left(\beta e^{-d_{1} \tau} \xi I^{*}+d+(1-a) r+\theta\right)(t-\rho \tau-\tau)} \\
& \times\left[S(\rho \tau+\tau)+(b-a r)(K-\epsilon)+\mu_{1} I^{*}\right] \\
& \times \int_{\rho \tau+\tau}^{t} e^{-\left(\beta e^{-d_{1} \tau} \xi I^{*}+d+(1-a) r+\theta\right)(t-\rho \tau-\tau)} d \theta \\
> & \frac{\left[(b-a r)(K-\epsilon)+\mu_{1} I^{*}\right]}{\beta e^{-d_{1} \tau} \xi I^{*}+d+(1-a) r+\theta} \\
& \times\left(1-e^{-\left(\beta e^{-d_{1} \tau} \xi I^{*}+d+(1-a) r+\theta\right)(t-\rho \tau-\tau)}\right),
\end{aligned}
$$

which gives us, for $t \geq 2 \rho \tau+\tau$,

$$
S(t)>S^{\Delta}>S^{*} \text {. }
$$

For $t \geq 0$, we define a positive differentiable function $V(t)$ as follows:

$$
V(t)=I(t)+\frac{[\beta(K-p)-\delta] e^{-d_{1} \tau}}{R_{0}} \int_{t-\tau}^{t} I(s) d s .
$$

We obtain the inequality, for $t \geq 2 \rho \tau+\tau$,

$$
\begin{aligned}
\dot{V}(t)= & {\left[\beta e^{-d_{1} \tau}\left(S(t)-S^{*}\right) I(t-\tau)+(1-a) r\right] } \\
& \times\left(1-\frac{N(t)}{K}\right) I(t) \\
> & \beta e^{-d_{1} \tau}\left(S(t)-S^{*}\right) I(t-\tau) \\
> & \beta e^{-d_{1} \tau}\left(S^{\Delta}-S^{*}\right) I(t-\tau) .
\end{aligned}
$$

Let $\underline{i}=\min _{\theta \in[-\tau, 0]} I(2 \rho \tau+2 \tau+\theta)$. Now, let us show that $I(t) \geq i$ for all $t \geq 2 \rho \tau+\tau$. In fact, if there exists $T_{2} \geq 0$ such that $I(t) \geq i$ for $2 \rho \tau+\tau \leq t \leq 2 \rho \tau+2 \tau+T_{2}, I\left(2 \rho \tau+2 \tau+T_{2}\right)=i$ and $\dot{I}\left(2 \rho \tau+2 \tau+T_{2}\right) \leq 0$. Direct calculation using the second equation of (1) and (48) gives

$$
\begin{aligned}
\dot{I}(2 \rho \tau+ & \left.2 \tau+T_{2}\right) \\
> & {\left[\beta e ^ { - d _ { 1 } \tau } \left(S\left(2 \rho \tau+2 \tau+T_{2}\right)\right.\right.} \\
& \left.-\left(d+(1-a) r+\mu_{1}+e^{-d_{1} \tau} \delta\right)\right] \underline{i} \\
> & \left(d+(1-a) r+\mu_{1}+e^{-d_{1} \tau} \delta\right)\left[\frac{S^{\Delta}}{S^{*}}-1\right] \underline{i}>0 .
\end{aligned}
$$

This contradicts the definition of $T_{2}$. Thus, we have shown that $I(t) \geq \underline{i}$ for all $t \geq 2 \rho \tau+\tau$. Hence, for all $t \geq 2 \rho \tau+2 \tau$,

$$
\dot{V}(t)>\beta e^{-d_{1} \tau}\left(S^{\Delta}-S^{*}\right) \underline{i},
$$

which implies that $V(t) \rightarrow+\infty$ as $t \rightarrow+\infty$. This contradicts the boundedness of $V(t)$. Consequently, $I\left(t_{0}\right)>$ $\xi I^{*}$ for some $t_{0} \geq \rho \tau$.

In the rest, we now need to consider two cases:

(i) $I(t) \geq \xi I^{*}$ for all large $t$;

(ii) $I(t)$ oscillates about $\xi I^{*}$ for all large $t$. 
We now need to show that $I(t) \geq \xi_{\mathcal{C}_{2}}$ for larget. Obviously, it suffices to show that it holds only for case (ii). We suppose that for any large $T$ there exists $t_{1}, t_{2}>T$ such that $I\left(t_{1}\right)=$ $I\left(t_{2}\right)=\xi I^{*}$ and $I(t)<\xi I^{*}$ for $t_{1}<t<t_{2}$. If $t_{2}-t_{1} \leq \tau$, the second equation (1) gives us $\dot{I}(t)>-\left(d+(1-a) r+\mu_{1}\right) I(t)$, which implies that $I(t)>I\left(t_{1}\right) e^{-\left(d+(1-a) r+\mu_{1}\right)\left(t-t_{1}\right)}$ on $\left(t_{1}, t_{2}\right)$. Thus, $I(t)>\xi_{\mathcal{C}_{2}}$. On the other hand, if $t_{2}-t_{1}>\tau$, applying the same manner gives $I(t) \geq \xi_{\mathcal{C}_{2}}$ on $\left[t_{1}, t_{1}+\tau\right]$, and hence the remaining work is to show $I(t) \geq \xi \mathcal{C}_{2}$ on $\left[t_{1}+\tau, t_{2}\right]$. In fact, assuming that there exists $T_{3}>0$ such that $I(t) \geq \xi_{c_{2}}$ on $\left[t_{1}, t_{1}+\tau+T_{3}\right], I\left(t_{1}+\tau+T_{3}\right)=\xi \mathcal{c}_{2}$, and $\dot{I}\left(t_{1}+\tau+T_{3}\right) \leq 0$, it follows from (1) that

$$
\begin{aligned}
\dot{I}\left(t_{1}+\tau+T_{3}\right) & \\
\geq & {\left[\beta e^{-d_{1} \tau} S\left(t_{1}+\tau+T_{3}\right)\right.} \\
& \left.-\left(d+(1-a) r+\mu_{1}+e^{-d_{1} \tau} \delta\right)\right] \xi_{\mathcal{C}_{2}} \\
> & \left(d+(1-a) r+\mu_{1}+e^{-d_{1} \tau} \delta\right)\left[\frac{S^{\Delta}}{S^{*}}-1\right] \xi_{\mathcal{C}_{2}}>0 .
\end{aligned}
$$

This contradicts the definition of $T_{3}$. Hence, $I(t) \geq \xi_{\mathcal{C}_{2}}$ on $\left[t_{1}, t_{2}\right]$. Consequently, $I(t) \geq \xi_{\mathcal{C}_{2}}$ for large $t$ in the case (ii). Therefore, $\lim _{t \rightarrow \infty} \inf I(t) \geq \xi c_{2}$. Note that $q$ may be so close to 1 that $\lim _{t \rightarrow \infty}$ inf $I(t) \geq c_{2}$.

Finally, let us show that $\lim _{t \rightarrow \infty} \inf R(t) \geq\left(\delta e^{-d_{1} \tau} c_{2}+\right.$ $\left.\theta c_{1}\right) /(d+(1-a) r)$. The third equation gives us

$$
\begin{aligned}
\dot{R}(t) & \geq\left[\delta e^{-d_{1} \tau} I+\theta S-[d+(1-a) r] R\right. \\
& \geq\left[\delta e^{-d_{1} \tau} \xi_{C_{2}}+\theta \xi c_{1}-[d+(1-a) r] R\right.
\end{aligned}
$$

for large $t$. Hence, $\lim _{t \rightarrow \infty} \inf R(t) \geq\left(\delta e^{-d_{1} \tau} \xi_{\mathcal{C}_{2}}+\right.$ $\left.\theta \xi_{c_{1}}\right) /(d+(1-a) r)$. In a similar manner, we could show $\lim _{t \rightarrow \infty} \inf R(t) \geq c_{3}$. This proves the theorem.

Corollary 14. If $\mu_{1}<\mu_{1}^{*}$ and $\theta<\theta^{*}$, then the system (1) with $\tau=\omega$ is permanent.

\section{Numerical Analysis}

Since it is important to visualize the dynamical behavior of the model, the model system (3) is integrated numerically with the help of MATLAB 7.0 using the following set of parameters.

(1) Let $r=0.5, k=8, d=0.04, d_{1}=0.04, \beta=1$, $a=0.3, \delta=0.2, \mu_{1}=0.8, \theta=0.01$, and $\tau=5$. It is easy to compute that $E_{0}=(8,0,0.2)$ and $R_{0}=0.94<1$. In Figure 1, the infective population and recovered population, respectively, are plotted against the total population. We see from the figure that for any initial start the solution curves tend to the equilibrium $E_{0}$. Hence, we infer that the system (3) may be stable about the disease-free equilibrium point $E_{0}$, which satisfies Theorem 9 .

(2) Let $r=0.5, k=8, d=0.04, d_{1}=0.04, \beta=1$, $a=0.3, \delta=0.2, \mu_{1}=0.2, \theta=0.02$, and $\tau=5$. We get $E^{*}=(8,2.23,1.11)$ and this set of parameter values satisfies the local asymptotic stability conditions of $E^{*}$. It is

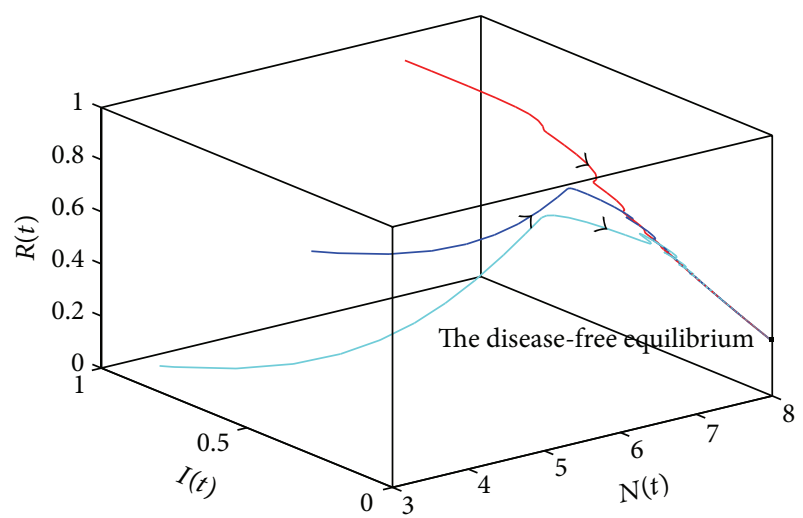

FIGURE 1: The disease-free equilibrium $E_{0}$ is locally asymptotically stable. Variation of infective population $I(t)$ and recovered population $R(t)$ with total population $N(t)$.

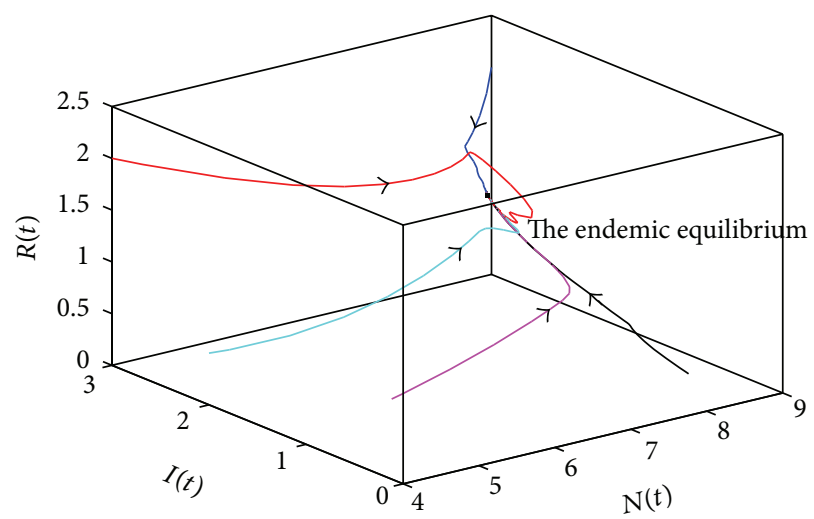

FIgURE 2: The endemic equilibrium $E^{*}$ is locally asymptotically stable. Variation of infective population $I(t)$ and recovered population $R(t)$ with total population $N(t)$.

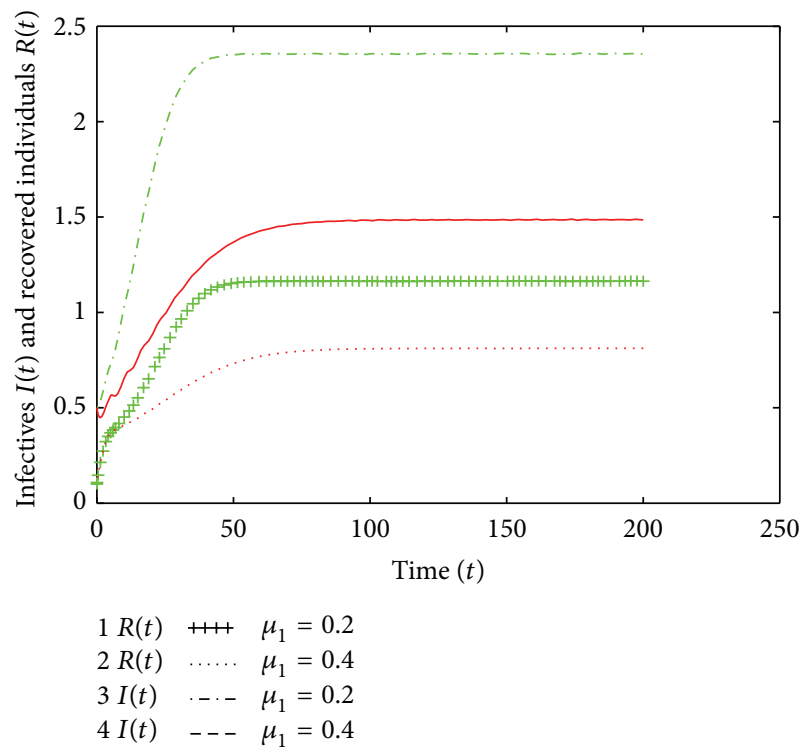

FIGURE 3: Variation of infective population $I(t)$ and recovered population $R(t)$ with time for different values of $\mu_{1}$. 


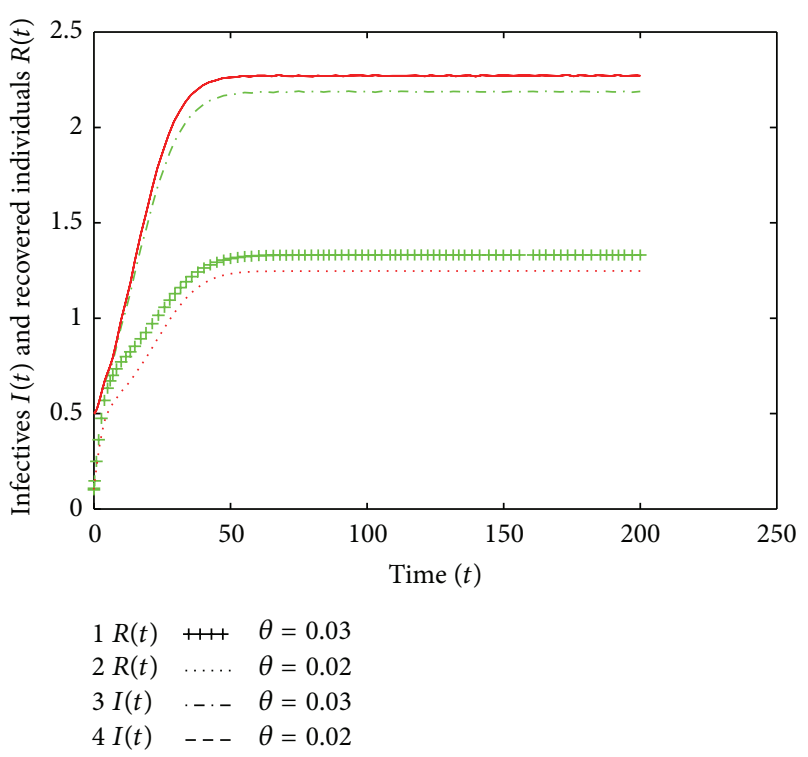

FIGURE 4: Variation of infective population $I(t)$ and recovered population $R(t)$ with time for different values of $\theta$.

easy to verify that $R_{0}=1.83>1$ and all other conditions of Theorem 11 are satisfied. So, we can obtain from Figure 2 that the system (3) is stable at the endemic equilibrium point $E^{*}$.

(3) The results of numerical simulation are displayed graphically in Figures 3 and 4. In Figure 3, the variation of the infective population and recovered population is shown with time for different values of the removal rate constant from groups $I$ to $S, \mu_{1}$. It is found that both the infective population and the recovered population decrease as $\mu_{1}$ increases. Figure 4 depicts the variation of infective population and recovered population, respectively, with time for the different successive vaccination rate, $\theta$. As $\theta$ increases, the infective population decreases whereas the recovered population increases.

\section{Discussion}

In this paper, we will consider two different delays which are important parameters on the dynamic behavior. So, the present study is continuation of the previous work by [43]. Furthermore, from biological epidemic point of view, we investigate successive vaccination and difference in immunity in our system. From mathematical point of view, we study the stability of disease-free equilibrium and the existence of endemic equilibrium for different delay and consider the permanence of the system in the new paper.

In Theorems 9, 10, 11, and 13 corresponding to their corollaries, when the effect of the successive vaccination rate and the transfer rate from the infectious group to the susceptible group after treatment is strong, that is, $\theta>\theta^{*}$ and $\mu_{1}>\mu_{1}^{*}$, the basic reproduction number $R_{0}$ being unity is a strict threshold for the control of the disease; the disease will be extinct or otherwise will tend to break out and persist. The other results are displayed graphically from our numerical simulation. We show the variation of the infective population and recovered population with time for different values of $\mu_{1}$. It is found that both the infective population and the recovered population decrease as $\mu_{1}$ increases. The infective population decreases whereas the recovered population increases as the successive vaccination rate increases, $\theta$, respectively.

\section{Conflict of Interests}

The authors declare that there is no conflict of interests regarding the publication of this paper.

\section{Acknowledgment}

This work is supported by National Natural Science Foundation of China (11101305).

\section{References}

[1] S. Ruan, W. Wang, and S. A. Levin, "The effect of global travel on the spread of SARS," Mathematical Biosciences and Engineering, vol. 3, no. 1, pp. 205-218, 2006.

[2] W. Wang and S. Ruan, "Simulating the SARS outbreak in Beijing with limited data," Journal of Theoretical Biology, vol. 227, no. 3, pp. 369-379, 2004.

[3] S. M. Moghadas and A. B. Gumel, "Global stability of a twostage epidemic model with generalized non-linear incidence," Mathematics and Computers in Simulation, vol. 60, no. 1-2, pp. 107-118, 2002.

[4] X. Zhou, X. Song, and X. Shi, "Analysis of stability and Hopf bifurcation for an HIV infection model with time delay," Applied Mathematics and Computation, vol. 199, no. 1, pp. 23-38, 2008.

[5] A. Tripathi, R. Naresh, and D. Sharma, "Modelling the effect of screening of unaware infectives on the spread of HIV infection," Applied Mathematics and Computation, vol. 184, no. 2, pp. 10531068, 2007.

[6] N. Yoshida and T. Hara, "Global stability of a delayed SIR epidemic model with density dependent birth and death rates," Journal of Computational and Applied Mathematics, vol. 201, no. 2, pp. 339-347, 2007.

[7] X. Meng and L. Chen, "The dynamics of a new SIR epidemic model concerning pulse vaccination strategy," Applied Mathematics and Computation, vol. 197, no. 2, pp. 582-597, 2008.

[8] A. d'Onofrio, "On pulse vaccination strategy in the SIR epidemic model with vertical transmission," Applied Mathematics Letters, vol. 18, no. 7, pp. 729-732, 2005.

[9] F. H. Chen, "A susceptible-infected epidemic model with voluntary vaccinations," Journal of Mathematical Biology, vol. 53, no. 2, pp. 253-272, 2006.

[10] I. A. Moneim and D. Greenhalgh, "Threshold and stability results for an SIRS epidemic model with a general periodic vaccination strategy," Journal of Biological Systems, vol. 13, no. 2, pp. 131-150, 2005.

[11] E. Shim, Z. Feng, M. Martcheva, and C. Castillo-Chavez, "An age-structured epidemic model of rotavirus with vaccination," Journal of Mathematical Biology, vol. 53, no. 4, pp. 719-746, 2006.

[12] G. Zaman, Y. H. Kang, and I. H. Jung, "Optimal treatment of an SIR epidemic model with time delay," BioSystems, vol. 98, no. 1, pp. 43-50, 2009. 
[13] Y. Pei, S. Liu, C. Li, and L. Chen, "The dynamics of an impulsive delay SI model with variable coefficients," Applied Mathematical Modelling, vol. 33, no. 6, pp. 2766-2776, 2009.

[14] S. Liu, Y. Pei, C. Li, and L. Chen, "Three kinds of TVS in a SIR epidemic model with saturated infectious force and vertical transmission," Applied Mathematical Modelling, vol. 33, no. 4, pp. 1923-1932, 2009.

[15] F. Brauer, "Epidemic models with heterogeneous mixing and treatment," Bulletin of Mathematical Biology, vol. 70, no. 7, pp. 1869-1885, 2008.

[16] S. Gao, L. Chen, and L. Sun, "Dynamic complexities in a seasonal prevention epidemic model with birth pulses," Chaos, Solitons \& Fractals, vol. 26, no. 4, pp. 1171-1181, 2005.

[17] Y. Pei and S. Liu, "Pulse vaccination strategy in a delayed SIRS epidemic model," in Proceedings of the 6th Conference of Biomathematics, Advanced in Biomathematics, vol. 2, pp. 775$778,2008$.

[18] T. Zhang and Z. Teng, "Permanence and extinction for a nonautonomous SIRS epidemic model with time delay," Applied Mathematical Modelling, vol. 33, no. 2, pp. 1058-1071, 2009.

[19] L. Nie, Z. Teng, L. Hu, and J. Peng, "Permanence and stability in non-autonomous predator-prey Lotka-Volterra systems with feedback controls," Computers \& Mathematics with Applications, vol. 58, no. 3, pp. 436-448, 2009.

[20] Y. N. Kyrychko and K. B. Blyuss, "Global properties of a delayed SIR model with temporary immunity and nonlinear incidence rate," Nonlinear Analysis: Real World Applications, vol. 6, no. 3, pp. 495-507, 2005.

[21] Y. Baba, Y. Tsuboi, H. Inoue, T. Yamada, Z. K. Wszolek, and D. F. Broderick, "Acute measles encephalitis in adults," Journal of Neurology, vol. 253, no. 1, pp. 121-124, 2006.

[22] A. Schreurs, E. V. Stålberg, and A. R. Punga, "Indication of peripheral nerve hyperexcitability in adult-onset subacute sclerosing panencephalitis (SSPE)," Neurological Sciences, vol. 29, no. 2, pp. 121-124, 2008.

[23] O. D. Makinde, "Adomian decomposition approach to a SIR epidemic model with constant vaccination strategy," Applied Mathematics and Computation, vol. 184, no. 2, pp. 842-848, 2007.

[24] D. Greenhalgh, Q. J. A. Khan, and F. I. Lewis, "Hopf bifurcation in two SIRS density dependent epidemic models," Mathematical and Computer Modelling, vol. 39, no. 11-12, pp. 1261-1283, 2004.

[25] S. Gao, L. Chen, and Z. Teng, "Impulsive vaccination of an SEIRS model with time delay and varying total population size," Bulletin of Mathematical Biology, vol. 69, no. 2, pp. 731-745, 2007.

[26] Z. Lu, X. Chi, and L. Chen, "The effect of constant and pulse vaccination on SIR epidemic model with horizontal and vertical transmission," Mathematical and Computer Modelling, vol. 36, no. 9-10, pp. 1039-1057, 2002.

[27] A. d'Onofrio, "On pulse vaccination strategy in the SIR epidemic model with vertical transmission," Applied Mathematics Letters, vol. 18, no. 7, pp. 729-732, 2005.

[28] G. Röst and J. Wu, "SEIR epidemiological model with varying infectivity and infinite delay," Mathematical Biosciences and Engineering, vol. 5, no. 2, pp. 389-402, 2008.

[29] M. Y. Li, H. L. Smith, and L. Wang, "Global dynamics an SEIR epidemic model with vertical transmission," SIAM Journal on Applied Mathematics, vol. 62, no. 1, pp. 58-69, 2001.

[30] S. A. Gourley, Y. Kuang, and J. D. Nagy, "Dynamics of a delay differential equation model of hepatitis B virus infection," Journal of Biological Dynamics, vol. 2, no. 2, pp. 140-153, 2008.
[31] J. Arino, J. R. Davis, D. Hartley, R. Jordan, J. M. Miller, and P. van den Driessche, "A multi-species epidemic model with spatial dynamics," Mathematical Medicine and Biology, vol. 22, no. 2, pp. 129-142, 2005.

[32] V. Capasso and G. Serio, "A generalization of the KermackMcKendrick deterministic epidemic model," Mathematical Biosciences, vol. 42, no. 1-2, pp. 43-61, 1978.

[33] S. Ruan and W. Wang, "Dynamical behavior of an epidemic model with a nonlinear incidence rate," Journal of Differential Equations, vol. 188, no. 1, pp. 135-163, 2003.

[34] W. M. Liu, S. A. Levin, and Y. Iwasa, "Influence of nonlinear incidence rates upon the behavior of SIRS epidemiological models," Journal of Mathematical Biology, vol. 23, no. 2, pp. 187204, 1986.

[35] S. Liu, Y. Pei, C. Li, and L. Chen, "Three kinds of TVS in a SIR epidemic model with saturated infectious force and vertical transmission," Applied Mathematical Modelling, vol. 33, no. 4, pp. 1923-1932, 2009.

[36] W. R. Derrick and P. van den Driessche, "A disease transmission model in a nonconstant population," Journal of Mathematical Biology, vol. 31, no. 5, pp. 495-512, 1993.

[37] A. Korobeinikov and P. K. Maini, "Non-linear incidence and stability of infectious disease models," Mathematical Medicine and Biology, vol. 22, no. 2, pp. 113-128, 2005.

[38] S. M. Moghadas and M. E. Alexander, "Bifurcations of an epidemic model with non-linear incidence and infectiondependent removal rate," Mathematical Medicine and Biology, vol. 23, no. 3, pp. 231-254, 2006.

[39] X. Ji, Y. Pei, and C. Li, "Two patterns of recruitment in an epidemic model with difference in immunity of individuals," Nonlinear Analysis: Real World Applications, vol. 11, no. 3, pp. 2078-2090, 2010.

[40] P. van den Driessche, "Time delay in epidemic models", in Mathematical Approaches for Emerging and Reemerging Infectious Diseases: An Introduction, C. Castillo-Chavez, S. Blower, P. van den Driessche, D. Kirschner, and A. Yakubu, Eds., vol. 126 of The IMA Volumes in Mathematics and its Applications, pp. 119-128, Springer, New York, NY, USA, 2002.

[41] H. W. Hethcote and P. van den Driessche, "Two SIS epidemiologic models with delays," Journal of Mathematical Biology, vol. 40, no. 1, pp. 3-26, 2000.

[42] W. Ma, Y. Takeuchi, T. Hara, and E. Beretta, "Permanence of an SIR epidemic model with distributed time delays," The Tohoku Mathematical Journal, vol. 54, no. 4, pp. 581-591, 2002.

[43] R. Naresh, A. Tripathi, J. M. Tchuenche, and D. Sharma, "Stability analysis of a time delayed SIR epidemic model with nonlinear incidence rate," Computers \& Mathematics with Applications, vol. 58, no. 2, pp. 348-359, 2009.

[44] H. Smith, An Introduction to Delay Differential Equations with Applications to the Life Sciences, vol. 57, Springer, New York, NY, USA, 2011. 


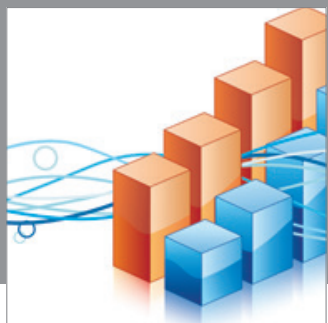

Advances in

Operations Research

mansans

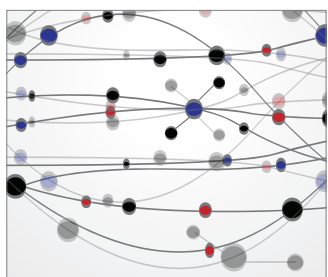

The Scientific World Journal
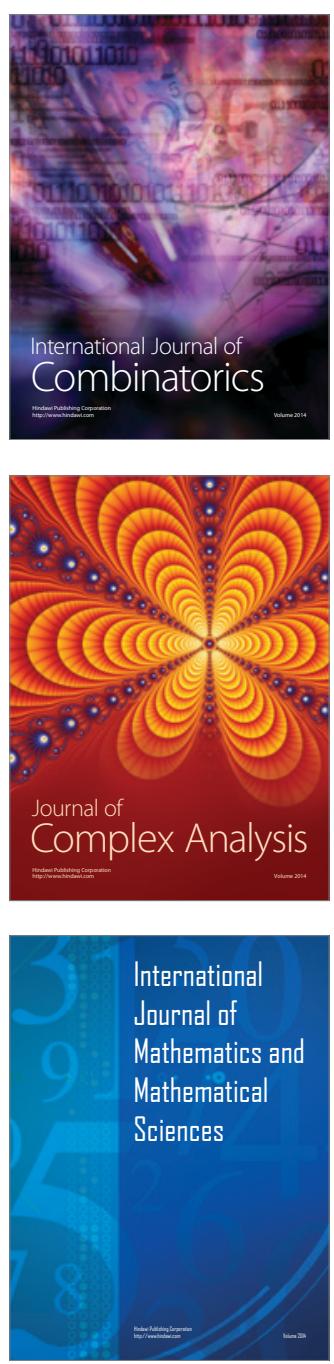
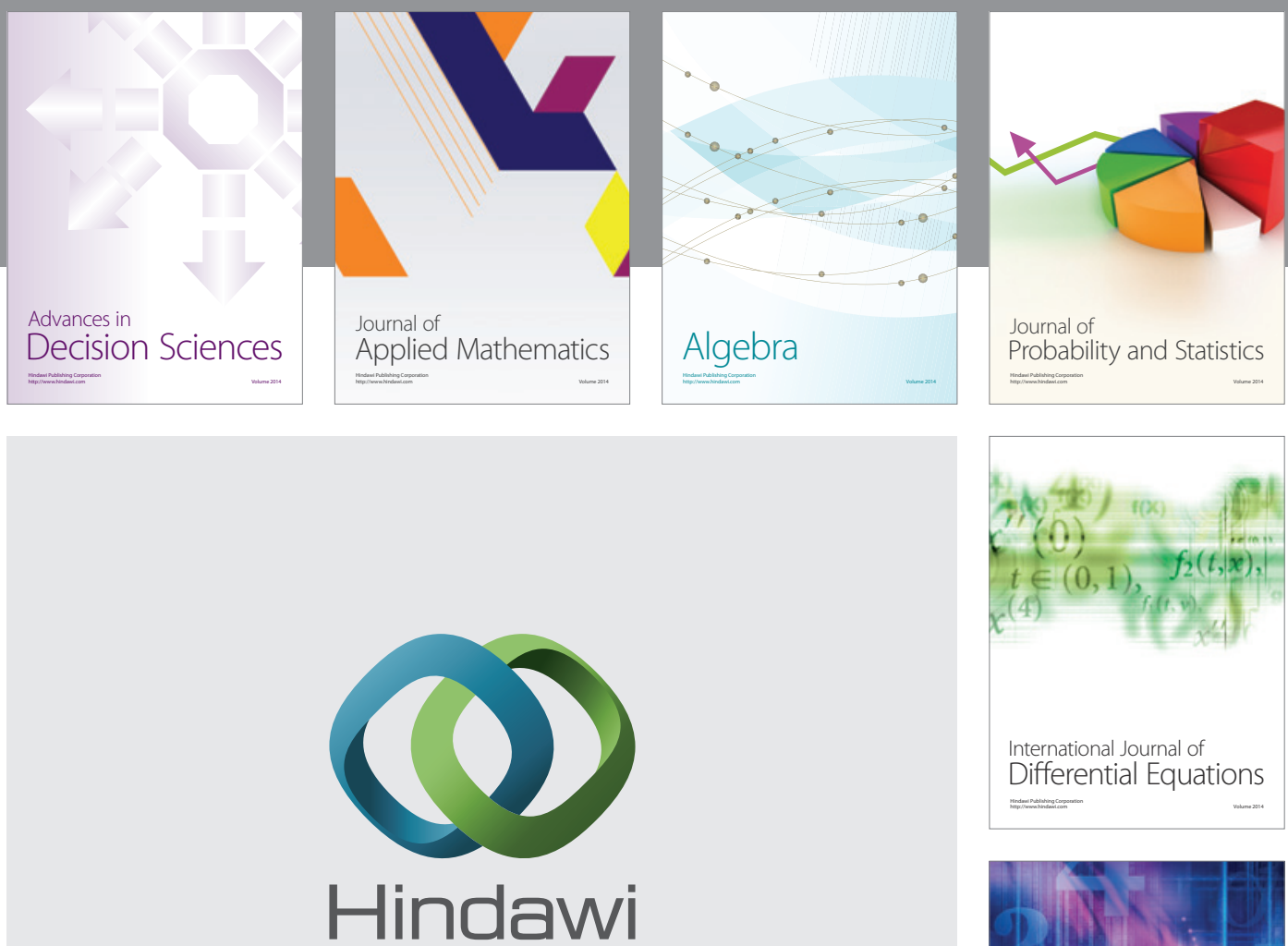

Submit your manuscripts at http://www.hindawi.com
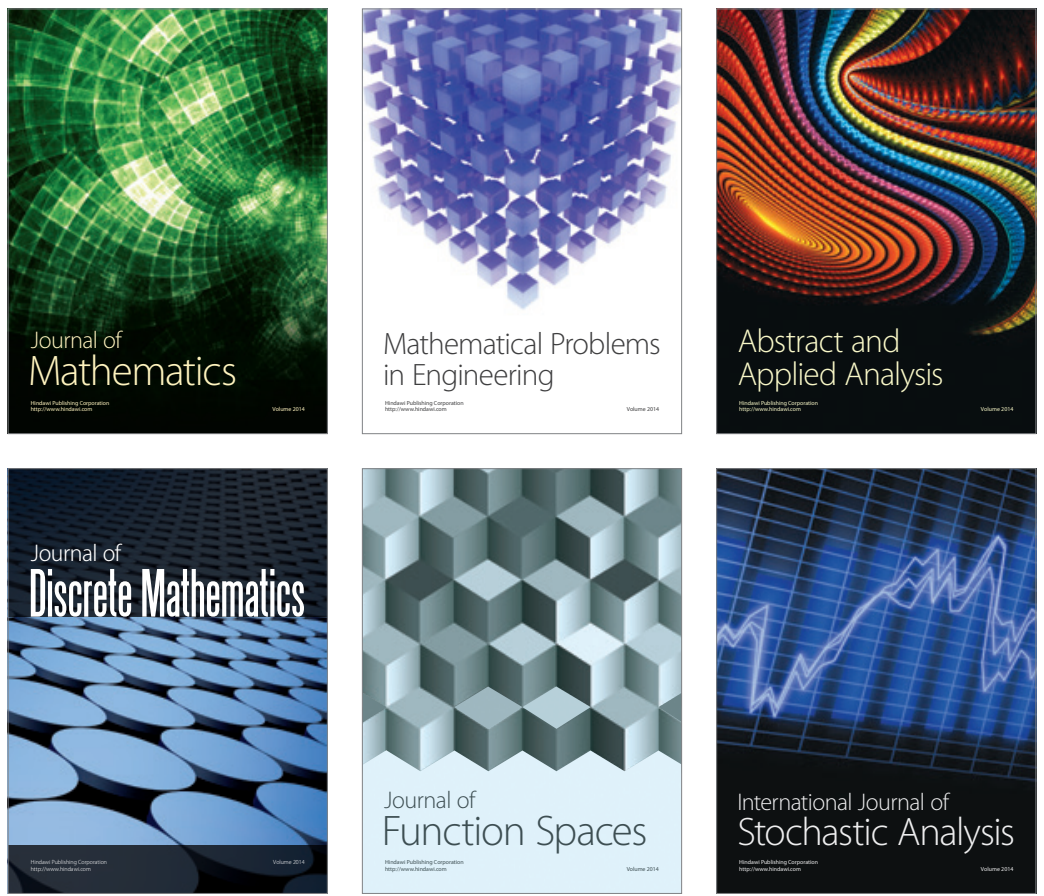

Journal of

Function Spaces

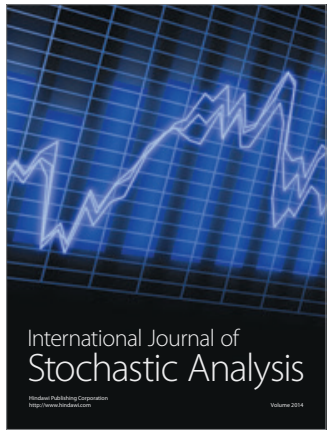

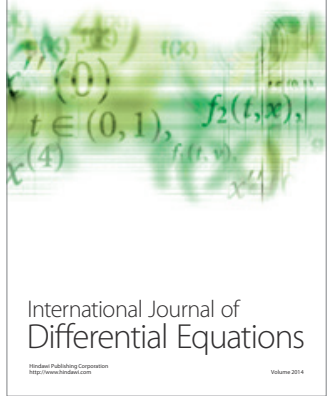
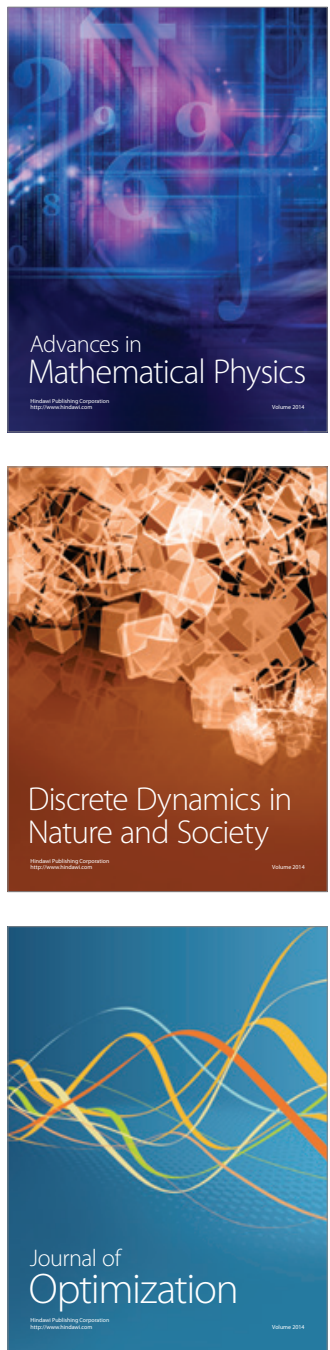